\title{
BUYING SEVERAL INDIVISIBLE GOODS
}

\author{
CARMEN BEVIA \\ MARTINE QUINZII \\ JOSÉ A. SILVA
}

\section{IN DB}

Working Paper Series \#97-20

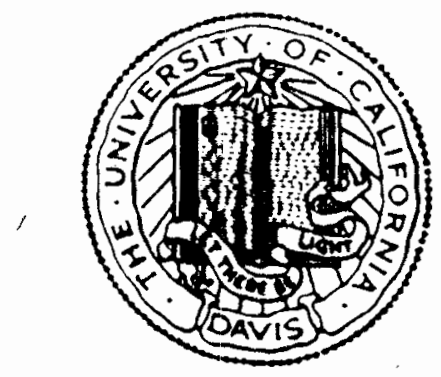

Department of Economics

University of California

Davis, California 95616-8578 


\title{
BUYING SEVERAL INDIVISIBLE GOODS
}

\author{
Carmen Bevia \\ University of Barcelona \\ Martine Quinzii \\ University of California, Davis \\ José A. Silva \\ University of Alicante
}

Working Paper Series No. 97-20

June, 1997

Note: The Working Papers of the Department of Economics, University of California, Davis, are preliminary materials circulated to invite discussion and critical comment. These papers may be freely circulated but to protect their tentative character they are not to be quoted without the permission of the author. 


\begin{abstract}
This paper studies economies where agents exchange indivisible goods and money. Agents have potential use for all indivisible goods and the indivisible goods are differentiated. We assume that agents have quasi-linear utilities in money, have sufficient money endowments to afford any group of objects priced below their reservation values, have reservation values which are submodular and satisfy the Cardinality Condition. This Cardinality Condition requires that for each agent the marginal utility of an object only depends on the number of objects to which it is added, not on their characteristics. Under these assumptions, we show that the set of competitive equilibrium prices is a non empty lattice and that, in any equilibrium, the price of an object is between the social value of the object and its value in its second best use.
\end{abstract}




\section{INTRODUCTION}

This paper considers economies where agents can buy and sell indivisible goods and in which all payments are made in units of a divisible good that, following standard use, we will call money. This model is probably closer to many circumstances of exchange in the real world than the standard model in which all goods are assumed to be perfectly divisible, but is also more difficult to analyze. The use of marginal calculus is precluded, and the application of fixed point theorems based on continuity properties, still possible in some cases, is certainly not straightforward. In consequence the model has beer! studied under restrictive assumptions, which are progressively being relaxed. Until recently it was assumed either that all the indivisible goods were all units of the same good (Henry (1970), or that buyers had use only for one type of indivisible good (Kaneko-Yamamoto (1986)), or just for one of the indivisible goods: this case covers most of the literature on assignment games and matching models (see Roth and Sotomayor for a comprehensive account of the results) and competitive equilibria of economies with indivisibilities (Kaneko (1982), Quinzii (1984), Gale (1984)). Recently several papers (Bikhchandani and Mamer (1994), Van der Laan, Talman and Yang (1995), Gul and Stacchetti (1996)) have relaxed this assumption, and assumed that agents have use for several units of the indivisible goods, units which may be differentiated.

With the exception of Bikhchandani and Mamer (1994) who show that, if there are two types of agents with quasilinear utilities such that all agents of the same type have the same supermodular and increasing reservation values, a competitive equilibrium exists, all the results of these papers follow from assumptions of the agents' demand functions rather than on their utility functions. Van der Laan and Talman (1995) impose a condition on agents' demands which seems to 
require separability of the utility functions with respect to the indivisible goods but not quasi-linearity in money, while Gul and Stacchetti impose that the utilities be quasi-linear in money and that the demands satisfy the property of Gross Substituability (to be formally defined in Section 2) introduced by Kelso and Crawford (1982) for a two-sided matching model between firms and workers. In contrast, this paper studies a class of economies which is defined by restrictions on the agents' utility functions. First, the utilities are quasi-linear in money so that the preferences can be represented by reservation values for subsets of the available indivisible objects. Second, these reservation values are submodular i.e. the marginal utility of an object decreases when the set of objects to which it is added becomes larger. Last and not the least, this marginal utility depends only on the number of objects and not on the composition of the set to which it is added.

This last assumption, that we call the Cardinality Condition, is certainly strong. At the moment however, it is the only interpretable condition that we have found which precludes that, for at least one agent, some objects "fit" better together than when they are associated with other objects - a situation which seems to cause nonexistence of an equilibrium even with decreasing marginal utilities (see the example of non-existence in Section 2 or the one in Gul and Stacchetti (1996)). Under the assumption that utilities are submodular and satisfy the Cardinality Condition we show that the set of equilibrium prices is non-empty, is a convex complete lattice and thus admits a vector $p^{M}$ of maximum and a vector $p_{m}$ of minimum equilibrium prices. Moreover these prices have a natural economic interpretation: the maximum price $p^{M}(\alpha)$ of an object a is the contribution of this object to the social welfare, or its social value, while its minimum price $p_{m}(\alpha)$ is its value in its second best use (these notions are precisely defined in Section 
3).

The proofs of the papers follow the route of the Second Welfare Theorem of Welfare Economics: we characterize the prices which support the efficient assignments of the objects, and use very few properties of agents' demands. In particular we do not use the property of Gross Substitutuability, which is the basis for the proofs of similar results by Gul and Stacchetti (1996), although this property is satisfied by the demands of agents whose utility functions satisfy our assumptions. Our proofs are thus alternative proofs to those of Gul and Stacchetti. Being based on the study of the efficient assignments of the objects, they uncover properties of these efficient assignments which are of interest in themselves, and may be used to obtain results of comparative statics for this class of economy (see in particular Lemma 3.3 which describes some regularities of the efficient assignments when new objects are added to the available objects)

The paper is organized as follows: the model and an example of nonexistence of equilibrium motivating the Cardinality Condition is presented in Section 2. The characterization of the prices supporting the efficient assignments of economies with submodular utility functions satisfying the Cardinality Condition is the subject of Section 3. Section 4 discusses the relation between the Cardinality Condition and the property of Gross Substitutability of demands.

\section{The Model}

Consider an exchange economy e with a finite set $\mathbf{I}$ of agents (whose elements are denoted by $\mathbf{i}, \mathbf{j}, \ldots$ ), a finiteset $\Omega$ of indivisible objects (whose elements are denoted by $\mathbf{a}, \beta, \ldots)$, and a perfectly divisible good called money. Agents' preferences are quasi-linear: the utility that agent $\mathbf{i} \in \mathbf{I}$ derives from consuming a set of objects $\boldsymbol{A}$ can be characterized by a reservation value $V(i, A)$ which represents the quantity 
of money that agent $\mathbf{i}$ is ready to sacrifice in order to consume the objects in A. The utility of agent $\mathbf{i}$ holding $m i$ units of money and the set $\mathrm{A}$ of objects is thus

$$
u_{i}\left(A, m_{i}\right)=V(i, A)+m_{i}
$$

For all $i \in \mathrm{I}$, the reservation value function $V(i,$.$) , defined on the power set$ $P(\Omega)$, is assumed to be weakly increasing $(V(i, A) \leq V(i, \mathrm{~B})$ whenever A C B $)$ and to satisfy $V(i, 0)=0$.

Agents' endowments, $\left(\bar{A}_{i}, \bar{m}_{i}\right)_{i \in I}$, with $\overline{m_{i}} \geq 0$ and $\cup_{i \in I} \bar{A}_{i}=\Omega$, are assumed to be such that $\bar{m}_{i} \geq V(i, \Omega)$ for all $i \in I$. This assumption implies that whenever the price of a set $\mathbf{A}$ of objects is less than the reservation value $V(i, \mathbf{A})$, agent $i$ can afford to buy the objects in A.

Let $\mathcal{E}$ denote the set of economies satisfying th $\mathrm{t}_{\mathrm{i}}$ ? abnve conditions. ior an economy $e \in \mathcal{E}$ an assignment a of objects to agents is thus a partition of the objects among the agents. Let $\Sigma(I, \Omega)$ denote all possible assignments. A feasible allocation of $e$ is a pair $(\mathrm{a}, \mathrm{m}) \in \Sigma(I, \Omega) \times R_{+}^{|I|}$ such that $\sum_{i \in I} m_{i}=\sum_{i \in I} \bar{m}_{i}$.

A Pareto optimal allocation is a feasible allocation $(a, m)$ such that there does not exist any other feasible allocation weakly preferred by all agents and strictly preferred by at least one agent. Of special interest in quasi-linear economies in which endowments of money are such that the nonnegativity constraints on money holdings never bind, are the Pareto ortimal allocations in which all agents' consumption of money is positive. These are the only Pareto optimal allocations which can possibly be obtained as competitive equilibrium allocations. As is well known, they are found by maximizing the sum of agents' utilities subject to the feasibility constraints, In this model they are the feasible allocations associated to the assignments which maximize the sum of the agents' reservation values. 
Such an assignment $\sigma$, satisfying

$$
\sum_{i \in I} V(i, \sigma(i)) \geq \sum_{i \in I} V(i, \tau(i)), \forall \tau \in \Sigma(I, \Omega)
$$

is called an efficient assignment.

Suppose that the objects are exchanged on a market at prices $(p(\alpha))_{\alpha \in \Omega}$. (Prices are expressed in units of money). If agent $i$ buys the set $\mathrm{A}$ of objects he will pay $p(A)=\sum_{\alpha \in A} p(\alpha)$. The demand of objects $D(i, p)$ of agent $\mathrm{i}$ at the price vector $\mathrm{p}=(p(\alpha))_{\alpha \in \Omega}$ is defined by

$$
D(i, p)=\{\mathrm{A} \in P(\Omega) \mid V(i, A)-p(A) \geq V(i, \mathrm{~B})-p(B), \forall B \in P(\Omega)\}
$$

The demand of agent $\mathrm{i}$ for money is then $m_{i}=\bar{m}_{i}+p\left(\bar{A}_{i}\right)-p(A)$. This number is always non negative since, for $A \in D(i, p), V(i, A)-p(A) \geq 0$ (the empty set is always a possible choice), and since by assumption $\bar{m}_{i} \geq V(i, \Omega)$. The price vector $\mathrm{p}$ is a competitive equilibrium price vector if, for each $i \in \mathbf{I}$, there exists $A_{i} \in D(i, p)$ such that the map $\mathrm{i}-\boldsymbol{A}$, is an assignment. By Walras Law, this condition, which ensures equilibrium on the market for the indivisible goods, implies that the market for money is also in equilibrium. Thus a competitive equilibrium for the economy $e$ can be defined as a pair $(\sigma, p) \in \Sigma(I, \Omega) \times R_{+}^{|\Omega|}$ such that $\sigma(i) \in D(i, p)$ for all $i \in \mathbf{I}$. It is easy to check that, if $(\sigma, p)$ is a competitive equilibrium, then a is an efficient assignment. We say that an efficient assignment $\sigma$ is supported by a price vector $\mathrm{p} \in R_{+}^{|\Omega|}$ if $(\sigma, p)$ is a competitive equilibrium.

Proposition 2.1. An economy e $\in \mathcal{E}$ has a competitive equilibrium if and only if every efficient assignment a of $\boldsymbol{e}$ can be supported by a price vector.

Proof. If $(\sigma, p)$ is a competitive equilibrium, by definition a is supported by $\mathrm{p}$. Suppose that 7 is another efficient assignment. Then

$$
V(i, \sigma(i))-p(\sigma(i)) \geq V(i, \tau(i))-p(\tau(i)), \forall i \in I
$$


The pair $(\tau, p)$ is not an equilibrium if at least one of the inequalities is strict. But then, summing the inequalities leads to

$$
\sum_{i \in I} V(i, \sigma(i))-p(\Omega)>\sum_{i \in I} V(i, \tau(i))-p(\Omega)
$$

which contradicts that $\tau$ is efficient. Thus $(\tau, p)$ is an equilibrium.

There exists a competitive equilibrium if and only if there is at least one efficient assignment supported by a price vector. By the above reasoning, this holds if and only if every efficient assignment is supported by a price vector.

The existence of an equilibrium is guaranteed (even without the assumption of quasi-linearity of preferences) if the agents have utility for at most one object $\left(V(i, A)=\max _{\alpha \in \Omega} V(i, \alpha)\right)$ ( Shapley-Shubik (1972), Kaneko (1982), Quinzii (1984)) or if all objects are identical $(V(i, A)=V(i,|A|)$ (Henry (1970)). In the case where agents can consume several indivisible objects which are not perfect substitutes for one another, an equilibrium exists, in the quasi-linear case that we are considering, if the reservation value functions are additive $(V(i, A)=$ $\left.\sum_{\alpha \in \Omega} V(i, a)\right)$. In this case it is efficient to give each object to the agent which values it most, and the price vector $\mathrm{p}$ such that $p(\alpha)=\max _{i \in I} V(i, \alpha)$ supports such an assignment. The object of this paper is to study the case where the objects are differentiated but where agents have use for more than one object. In this case an equilibrium may not exist (Henry (1970), Bikchandani-Mamer (1996)) and additional restrictions must be placed on the reservation value functions.

A condition that seems particularly attractive since it expresses, in the case of indivisible goods, the idea that the marginal utility of an additional item decreases when the bundle of goods to which it is added gets larger, is the assumption of submodularity. 
Definition 2.2. A reservation value function is said to be submodular if it is satisfies for all $A, B$ in $P(\Omega)$

$$
V(i, A \cup B) \leq V(i, A)+V(i, B)-V(i, A \cap B)
$$

or equivalently

$$
V(i, A)-V(i, A \backslash \alpha) \leq V(i, B)-V(i, B \backslash \alpha), \text { for all } \alpha \in B \subseteq A
$$

Unfortunately this assumption is not sufficient to imply existence of an equilibrium, as shown by the following example.

Example 1. Let e $\in \mathcal{E}$ be such that $\mathbf{I}=\{1,2,3\}, \Omega=\{\alpha, \beta, \gamma\}$. The submodular reservation values of the agents for the different subsets of objects are given in the following table

\begin{tabular}{|l|l|l|l|l|l|l|l|}
\hline$V \backslash A$ & $\alpha$ & $\beta$ & $\gamma$ & $\alpha \beta$ & $\alpha \gamma$ & $\beta \gamma$ & $\alpha \beta \gamma$ \\
\hline$V(1, A)$ & 10 & 8 & 2 & 13 & 11 & 9 & 14 \\
$V(2, A)$ & 8 & 5 & 10 & 13 & 14 & 13 & 15 \\
$V(3, A)$ & 1 & 1 & 8 & 2 & 9 & 9 & 10 \\
\hline
\end{tabular}

The only efficient assignment $a$ of objects in this economy is such that $\sigma(1)=$ $\beta, \sigma(2)=\alpha, \sigma(3)=y$. Suppose that $p$ supports this assignment. In order that buying $\{\alpha \beta\}$ is not better for agent 2 than buying only $a$, p must be such that $p(\beta) \geq 5$. In order that buying no object is not better for agent 3 than buying $\gamma, p$ must be such that $p(\gamma) \leq 8$. In order that buying $a$ is not better for agent 1 than buying $\beta, p$ must be such that $p(\alpha) \geq p(\beta)+2$. In order that buying $\gamma$ is not better for agent 2 than buying $a, p$ must be such that $p(\gamma) \geq p(\alpha)+2$. Combining these inequalities gives $7 \leq 2+p(\beta) \leq p(a) \leq p(\gamma)-2 \leq 6$, which is impossible. 
Submodularity of the reservation values still permits complicated interdependence in utility among objects, which may prevent the existence of an equilibrium. In the previous example, if agent 2 has objects a and $\beta$, then the marginal contribution of $\mathbf{a}$ is equal to its value $\mathrm{V}(2, \mathrm{a})$ since $\mathrm{V}(2, \alpha \beta)-\mathrm{V}(2, \beta)=\mathbf{1 3}-\mathbf{5}=8=$ $V(2, \alpha)$, while if objects $\alpha$ and $\gamma$ are combined the marginal contribution of $a$ is much lower: $\mathrm{V}(2, \alpha \gamma)-V(2, \gamma)=\mathbf{1 4}-10<\mathrm{V}(2, \mathrm{a})$. Thus, for agent 2 , having object $\beta$ at the same time does not subtract any of the value of a while having $\gamma$ lowers the desirability of a .

A sufficient condition which ensures that the interdependence among objects is weak and that guarantees the existence of an equilibrium is that the demands of all agents satisfy the Gross Substitute (GS) assumption introduced by KelsoCrawford (1982). Heuristically the demand of agent i satisfies the GS condition if, when the price of an object - - let us say $\beta$-increases while the prices of all other objects stay the same, then the objects other than $\beta$ which were demanded by agent $i$ are still demanded by this agent. This implies that there is no object which was demanded by agent $i$ because it "fitted" especially well with $\beta$, but is no longer desirable when $\beta$ becomes too expensive. To state the formal definition of the GS property, let us adopt the following convention: we say that the object $\mathrm{a}$ in the demand of agent $i$ at prices $\mathrm{p}$ if it belongs to at least one subset of objects demanded by agent $i$ at $p$.

$$
\alpha \in D(i, p) \Leftrightarrow \alpha \in A \text { for some } A \in D(i, p)
$$

Definition 2.3. The demand of agent $i$ satisfies the Gross Substitute property if for any $\mathrm{p} \in R^{|\Omega|}$, and any $\tilde{p} \in R^{|\Omega|}, \tilde{p} \geq \mathrm{p}$, with $\tilde{p}(\alpha)=p(\alpha)$ for some a $\in \Omega$, then a $\in D(i, p)$ implies $\mathbf{a} \in D(i, \tilde{p})$.

The Gross Substitute property however is not a condition on the primitive 
characteristics of the economy (the utility functions $V(i, \cdot)$ ) but a condition on the derived demand functions (or more accurately demand correspondences). In this paper we will study a condition in the same spirit, which is stronger, but is made directly on the utility functions. The "Cardinality Condition" that we impose requires that the marginal contribution of an object only depends on the number of objects to which it is added. This condition prevent interactions in utilities among objects - like objects $\alpha$ and $\beta$ fitting especially well togetherwhich create problems for the existence of equilibrium.

Definition 2.4. The utility function! $V(i, \cdot)$ satisfies the Cardinality Condition if the marginal contribution of an object to agent i's utility depends only on the number of objects to which it is added, i.e., for all $A, \mathrm{~B} \in P(\Omega)$ such that $|A|=|B|$ and $\alpha \in A \cap B$

$$
V(i, A)-V(i, A \backslash \alpha)=V(i, B)-V(i, B \backslash \alpha)
$$

Assumption $C$ makes sense only for indivisible objects which have the same function, since otherwise the marginal utility of an object for an agent depends on the composition of the set of objects to which it is added, not just on its cardinality (in particular it depends on whether or not the agent already possesses an object performing the same function). However if there are indivisible objects of different nature and if the utility of the agents are additive between groups of objects of different nature, then each group of objects can be attributed (auctioned, sold) separately. Under the separability assumption, it is sufficient to study "two good" economies with money and indivisible objects which are all of the same nature. The model studied in this paper generalizes, in the quasi-linear case the two-good model studied by Henry (1n70) to the case where the indivisible objects are differentiated. An example of such objects could be paintings-or 
more generally art objects - collected by agents for purpose of decoration. The objects typically have different "esthetic values" for different agents, and if the agents are more sensitive to the effect of each object than to the general effect that a group of objects produce together, then the Cardinality Condition can be a reasonable approximation. The assumption that the agents' reservation values are submodular seems also reasonable in this case, unless some agents are obsessive collectors.

In the following section we prove that if the utility functions are submodular and satisfy the Cardinality Condition, the set of equilibrium prices has the lattice property found in the matching model:; with quasi-linear utilities. Thus if $p^{M}(\alpha)$ is the maximum value of object a for any equilibrium and $p_{m}(\alpha)$ its lowest value in any equilibrium, then the vectors $p^{M}$ and $p_{m}$ are also equilibrium prices. Of course $p^{M}$ is the vector of equilibrium prices which is the most favorable for the sellers and $p_{m}$ the most favorable for the buyers. Moreover the prices $p^{M}$ and $p_{m}$ have a natural economic interpretation: $p^{M}$ (a) is the social surplus created by object a (to be precisely defined in Section 3) while $p_{m}(\alpha)$ is the value of $\mathbf{a}$ in its second best use (also to be defined in Section 3).

Similar properties of the equilibrium prices have been independently derived in a recent paper by Gul and Stacchetti (1996) under the assumption that the demands of all agents satisfy the Gross Substitute property. However the proofs are different in nature from the proofs of this paper. In the quasi-linear economies with large endowments of money that we are considering the existence of equilibrium and the Second Theorem of Welfare economics (Pareto optimal allocations maximizing the sum of agents' utilities can be supported by prices) are equivalent, since the equilibrium prices do not depend on the income distribution (see Proposition 2.1). While Stacchetti and Gul call on the properties of the agents' 
demand functions to prove existence of an equilibrium and derive the properties of equilibrium prices, the analysis of this paper follows the route of the second Theorem of Welfare economics and shows that the prices $p^{M}$ and $\mathrm{p}^{\mathrm{m}}$ as well as a convex, complete lattice of intermediate prices support the efficient allocations of the objects among the agents.

\section{Equilibrium Prices}

\subsection{Definition of $p^{M}$ and $p_{m}$}

The analysis of this section is made under the following set of assumptions on the utility functions which will not be repeated

ASSUMPTION : For all $i \in \mathbf{I}$, the utility function $V(i, \cdot)$ is submodular and satisfy the Cardinality Condition

Let a be an efficient assignment of the objects $\Omega$ to the $\mathbf{I}$ agents. The goal of this section is to derive the prices supporting this allocation of the objects. In a model with divisible goods and quasi-linear utilities, the prices supporting a Pareto optimal allocation are given by the multipliers associated to the scarcity constraints in the program of maximization of the sum of the utilities (social welfare) subject to the feasibility constraints. The envelope theorem then permit interpreting the multiplier associated to the scarcity constraint for a good (let us say good a ) as the change in social welfare resulting from a marginal decrease or increase in the supply of this good. Suppose now that good a is indivisible and exists in a single unit. If we proceed by analogy, there are two changes in the supply of a which play the role of a marginal change in the supply of $a$ when the good is divisible: the supply can be decreased by one unit by taking the good a out of the available supply of goods; or the supply can be increased by one unit 
by adding a copy of a to the supply of available goods. These changes induce changes in social welfare analogous to the changes in social welfare accompanying a marginal change in the supply of a divisible good. We will prove that these changes in social welfare define the maximum and minimum prices supporting the efficient allocation $\sigma$.

Let us thus define the social welfare created by a supply $\Omega$ of objects by

$$
U(\Omega) \doteq \underset{i \in I}{ } V(i,(\mathrm{i})), \quad \text { for any efficient assignment } \sigma \text { of } \Omega
$$

Define $p^{M}$ as the change in social welfare when the object $a$ is taken out of the available objects, i.e.

$$
p^{M}(\alpha)=U(\Omega)-U(\Omega \backslash \alpha), \quad \alpha \in \Omega
$$

or alternatively as the contribution of a to the social welfare. To define the minimum prices, for all $\alpha \in \Omega$ let $\widetilde{\alpha}$ denote an exact copy of object $a$. To define the social welfare associated to $\Omega \cup \widetilde{\alpha}$, we need to extend the utility functions to subsets of $\Omega \mathrm{U} \widetilde{\alpha}$ containing both a and $\widetilde{\alpha}$. The extension is made in the following way: if $\mathrm{A}$ is a subset of $\Omega \cup \widetilde{\alpha}$ such that $\mathbf{a} \in \boldsymbol{A}, \widetilde{\alpha} \in \mathrm{A}$, then $V(i, \mathrm{~A})=V(i, A \backslash \widetilde{\alpha})$. Thus no agent benefits from having two copies of the same object. With this convention, there is always an efficient assignment of $\Omega \cup \widetilde{\alpha}$ which does not give a and $\widetilde{\alpha}$ to the same agent (even if only one agent has any use for a, as long as we assume free disposal). In all that follows, we will only consider efficient assignments $\mathrm{p}$ of $\Omega \cup \widetilde{\alpha}$ such that $\rho(i) \in \Omega$. In the normal case where several agents have a positive utility for a, an. efficient allocation of $\Omega \cup \widetilde{\alpha}$ defines the second best use of a, since it becomes possible to give $\alpha$ to the agent $\mathrm{i}$ who has it in an efficient allocation of $\Omega$, and to give a copy to the agent who would most benefit from a after agent $\mathbf{i}$ (see the Remark after Lemma 3.3). If we define 


$$
p_{m}(\alpha)=U(\Omega \cup \widetilde{\alpha})-U(\Omega), \alpha \in \Omega
$$

we can interpret $p_{m}(\alpha)$ as the social value of $a$ in its second best use. Define $p^{M}=\left(p^{M}(\alpha)\right)_{\alpha \in \Omega}$ and $p_{m}=\left(p_{m}(\alpha)\right)_{\alpha \in \Omega}$. We first show that $p^{M}(\alpha)$ and $p_{m}(\alpha)$ give respectively the highest and lowest possible equilibrium prices for object $a$.

Proposition 3.1. Let $a$ be an efficient assignment of $\Omega$, and suppose that there exists $p \in R_{+}^{|\Omega|}$ supporting the assignment $\sigma$. Then for all $a \in \Omega, U(\Omega \cup \widetilde{\alpha})-U(\Omega) \leq$ $p(\alpha) \leq U(\Omega)-U(\Omega \backslash \alpha)$

Proof. Since the vector $p$ is supporting the assignment $a$, for all $i \in I$, and for all $A \in P(\Omega), V(i, \sigma(i))-p(\sigma(i)) \geq V(i, A)-p(A)$. In particular, given $a \in \Omega$, lei $\tau$ be an efficient assignment of $\Omega \backslash \alpha$ among $I$, and let $p$ be an efficient assignment of $\Omega \cup \widetilde{\boldsymbol{\alpha}}$. As noted above, assume w.l.o.g. that $\rho(i) \in \Omega$ for all $i$. Then, $V(i, \sigma(i))-$ $p(\sigma(i)) \geq V(i, \tau(i))-p(\tau(i))$ and $V(i, \sigma(i))-p(\sigma(i)) \geq V(i, \rho(i))-p(\rho(i))$ for all $i \in I$. Summing up this inequalities, we get $U(\Omega)-p(\alpha) \geq U(\Omega \backslash \alpha)$ and $U(\Omega) \geq U(\Omega \cup \widetilde{\alpha})-p(\alpha)$

The next two lemmas will be frequently used in proving that $p^{M}$ and $p_{m}$ are equilibrium price vectors.

Lemma 3.2. Let $a$ and $\beta$ be two objects in $\Omega$ and let $C$ and $D$ be two subsets of $\Omega$ such that $\{\alpha, \beta\} \cap C=0$ and $\{a, \beta\} \cap D=0$. Then for all $i \in \mathbf{I}$

$$
V(i, C \cup \alpha)-V(i, C \cup \beta)=V(i, D \cup \alpha)-V(i, D \cup \beta)
$$

Proof. Suppose first that $|C|=|D|$. Then by the cardinality condition

$$
\begin{aligned}
& V(i, C \cup \alpha)-V(i, C)=V(i, D \cup \alpha)-V(i, D) \\
& V(i, C \cup \beta)-V(i, C)=V(i, D \cup \beta)-V(i, D)
\end{aligned}
$$


Subtracting the second equality from the first gives the result. Suppose now that $|C|=|D|-1$. Then $|C \cup \alpha|=|C \cup \beta|=|D|$. The cardinality condition then implies

$$
\begin{aligned}
& V(i, C \cup \alpha \cup \beta)-V(i, C \cup \alpha)=V(i, D \cup \beta)-V(i, D) \\
& V(i, C \cup \alpha \cup \beta)-V(i, C \cup \beta)=V(i, D \cup \alpha)-V(i, D)
\end{aligned}
$$

Subtracting the second inequality from the first gives the result. Suppose that $|C|=|D|-2$. Then consider a subset $D^{\prime} \mathrm{c}$ D such that $|C|=\left|D^{\prime}\right|-1$. Applying twice the previous step gives

$V(i, C \cup \alpha)-V(i, C \cup \beta)=V\left(i, D^{\prime} \cup \alpha\right)-V\left(i, D^{\prime} \cup \beta\right)=V(i, D \cup \alpha)-V(i, D \cup \beta)$

Thus the property holds if $|C| \leqslant|D|$. Since $C$ and $D$ play a symmetrical role, the property holds for all subsets $C$ and $D$ which do not contain $\alpha$ or $\beta$.

Lemma 3.3. Let $a$ be an efficient assignment of $\Omega$ to the agents. For any $a \in \Omega$ there is an efficient assignment $\tau$ of $\Omega^{\prime} \backslash \alpha$ and an efficient assignment $\rho$ of $\Omega \cup \widetilde{\alpha}$ such that $|\tau(i)| \leq|\sigma(i)| \leq|\rho(i)|$ for all $i \in I$. Moreover $\rho$ can be constructed such that the agent who has $a$ in the assignment $a$ also has it in the assignment $p$.

Proof. Step 1: Let $\tau$ be an efficient assignment of $\Omega \backslash \alpha$ among $I$. Partition the set $I$ of agents between the subsets

$I_{1}=\{i \in I|| \tau(i)|>| \sigma(i) \mid\}, \quad I=\{i \in I|\tau(i)|=|\sigma(i)|\}, \quad I_{3}=\{i \in I|| \tau(i)|<| \sigma(i) \mid\}$

and suppose that $I_{1}$ is not empty, that is for some $i \in I,|\tau(i)|>|\sigma(i)|$. Choose an agent $i \in I_{1}$. There exist $\beta \in \tau(i)$ such that $\beta \in \sigma(j)$ for some agent $j \neq i$.

Suppose first that $j \in I_{3}$, i.e. $|\tau(j)|<|\sigma(j)|$. Consider a new assignment $\tau^{*}$ where agent $j$ gets $\tau(j) \cup \beta$ and agent $i$ gets $\tau(i) \backslash \beta$. Since $|\tau(j)|<|\sigma(j)|$, 
$|\tau(j) u \beta| \leq|\sigma(j)|$, then by submodularity and Assumption $\mathrm{C}$ we obtain that

$$
V(j, \tau(j) \cup \beta)-V(j, \tau(j)) \geq V(j, \sigma(j))-V(j, \sigma(j) \backslash \beta)
$$

Since $a$ is an efficient assignment of $\Omega$ among $I$,

$$
V(j, \sigma(j))-V(j, \sigma(j) \backslash \beta) \geq V(i, \sigma(i) \cup \beta)-V(i, \sigma(i))
$$

Since $|\sigma(i)|<|\tau(i)|,|\sigma(i) \cup \beta| \leq|\tau(i)|$, and by submodularity and Assumption $\mathrm{C}$,

$$
V(i, \sigma(i) U \beta)-V(i, \sigma(i)) \geq V(i, \tau(i))-V(i, \tau(i) \backslash \beta)
$$

Therefore,

$$
V(j, \tau(j) \cup \beta)-V(j, \tau(j)) \geq V(i, \tau(i))-V(i, \tau(i) \backslash \beta)
$$

which proves that the new assignment of $\Omega \backslash \alpha$ is as efficient as I-. If the inequality is strict, this is a contradiction with the fact that $\tau$ is an efficient assignment of $\Omega \backslash \alpha$ among $I$. Thus there must be equality, and the new assignment $\tau^{*}$ obtained by shifting $\beta$ from agent $i$ to agent $j$ is an efficient assignment of $R a$ among $I$ which has decreased by one the number of objects attributed to the agents of $I_{1}$.

Suppose now that $|\tau(j)| \geq|\sigma(j)|$, that is $j \in I_{1} \cup I_{2}$. Then there exists an object $\beta_{1}$ in $\tau(j)$ which is not in $\sigma(j)$ and is thus in $\sigma\left(j_{1}\right)$ for some agent $j_{1}$. For symmetry of notation call $j_{0}$ the agent who has $\beta$ under $a\left(j=j_{0}\right)$ and call $\beta=\beta_{0}$. If $j_{1}$ is in $I_{3}$ then consider the assignment $\tau^{*}$ obtained by transferring $\beta_{0}$ to agent $j_{0}$ and $\beta_{1}$ to agent $j_{1}$. If agent $j_{1}$ is in $I_{1} \cup I_{2}$, then continue the procedure by finding an object $\beta_{2}$ in $\tau\left(j_{1}\right)$ which is not in $\sigma\left(j_{1}\right)$ and is thus in $\sigma\left(j_{2}\right)$ for some agent $j_{2} \cdots$ until an agent of $I_{3}$ is reached. If the same agent in $I_{1} \mathrm{U} I_{2}$ is selected at several stage of the procedure always choose a new object, so that $\beta_{0} \neq \beta_{1} \neq \beta_{2} \neq \cdots$. This is possible since for these agents $|\tau(i)| \geq|\sigma(i)|$ 
so that each object which is attributed to $i$ under a and not under $\tau$ has been replaced by a new object. Since $I_{3}$ is non empty, (there are less objects in $\Omega \backslash \alpha$ than in St) after a finite number of steps the procedure must stop by reaching an agent $j_{k}$ in $I_{3}$. Consider the assignment $\tau^{*}$ obtained by transferring $\beta_{0}$ from $i$ to $j_{0}, \beta_{1}$ from $j_{0}$ to $j_{1}, \cdot \cdot, \beta_{k}$ to $j_{k}$. Note that without loss of generality we can assume that the agents $j_{0}, j_{1}, \ldots, j_{k}$ are all different. For if the same agent is chosen at differentstage of the procedure, i.e. if, for $\ell \geq 0, r \geq 1, j_{\ell}=j_{\ell+r}$, then it is possible to choose directly the object $\beta_{\boldsymbol{\ell}+r+1}$ instead of $\beta_{\boldsymbol{\ell}+1}$ in $\tau\left(j_{\ell}\right)$ the first time that agent $j_{\ell}$ is reached, avoiding the cycle $j_{\ell}, j_{\ell+1}, \ldots, j_{\ell+r}$. Let us show that the assignment $\mathrm{T}^{*}$ is efficient. Applying Lemma 3.2 with $\mathrm{C}=\tau\left(j_{\ell}\right) \backslash \beta_{\ell+1}$ and $\mathrm{D}=\sigma\left(j_{\ell}\right) \backslash \beta_{\ell}$ gives for agents $j_{0}, \ldots, j_{k-1}$

$$
V\left(j_{\ell}, \tau\left(j_{\ell}\right) \backslash \beta_{\ell+1} \cup \beta_{\ell}\right)-V\left(j_{\ell}, \tau\left(j_{\ell}\right)\right)=V\left(j_{\ell}, \sigma\left(j_{\ell}\right)\right)-V\left(j_{\ell}, \sigma\left(j_{\ell}\right) \backslash \beta_{\ell} \cup \beta_{\ell+1}\right)
$$

For agent i, since $|\tau(i)|>|\sigma(i)|$, by the cardinality condition

$$
V(i, \sigma(i) \cup \mathrm{Do})-V(i, \sigma(i)) \geq V(i, \tau(i))-V\left(i, \tau(i) \backslash \beta_{0}\right)
$$

and for agent $j_{k}$, since $\left|\tau\left(j_{k}\right)\right|<\left|\sigma\left(j_{k}\right)\right|$

$$
V\left(j_{k}, \tau\left(j_{k}\right) \cup \beta_{k}\right)-V\left(j_{k}, \tau\left(j_{k}\right)\right) \geq V\left(j_{k}, \sigma\left(j_{k}\right)\right)-V\left(j_{k}, \sigma\left(j_{k}\right) \backslash \beta_{k}\right)
$$

Adding up these equalities and inequalities leads to

$$
\begin{aligned}
& {\left[V\left(i, \tau(i) \backslash \beta_{0}\right)+V\left(j_{0}, \tau\left(j_{0}\right) \backslash \beta_{1} \cup \beta_{0}\right)+\ldots+V\left(j_{k}, \tau\left(j_{k}\right) \cup \beta_{k}\right)\right] } \\
& -\left[V(i, \tau(i))+V\left(j_{0}, \tau\left(j_{0}\right)\right)+\ldots+V\left(j_{k}, \tau\left(j_{k}\right)\right)\right] \\
\geq \quad & {\left[V(i, \sigma(i))+V\left(j_{0}, \sigma\left(j_{0}\right)\right)+\ldots+V\left(j_{k}, \sigma\left(j_{k}\right)\right)\right] } \\
& -\left[V\left(i, \sigma(i) \cup \beta_{0}\right)+V\left(j_{0}, \sigma\left(j_{0}\right) \backslash \beta_{0} \cup \beta_{1}\right)+\ldots+V\left(j_{k}, \sigma\left(j_{k}\right) \backslash \beta_{k}\right)\right] \\
\geq & 0
\end{aligned}
$$


where the last inequality is implied by the efficiency of $\mathbf{a}$.

Thus as long as an efficient assignment $\tau$ of $\Omega \backslash \alpha$ is such that $I_{1}$ is not empty it is possible to construct another efficient assignment $\tau^{*}$ with one less object attributed to the agents of $I_{1}$ and one more to the agents of $I_{3}$. In a finite number of such steps we must find and efficient assignment $\tau$ of $\Omega \backslash \alpha$ such that $I_{1}$ is empty.

Step 2: Consider now an efficient assignment $\mathrm{p}$ of $\Omega \mathrm{U} \widetilde{\boldsymbol{\alpha}}$ and partition the set I into

$J_{1}=\{i \in I|| \sigma(i)|>| \rho(i) \mid\}, \quad J_{2}=\{i \in I|| \sigma(i)|=| \rho(i) \mid\}, \quad J_{3}=\{i \in I|| \sigma(i)|<| \rho(i) \mid\}$

If $J_{1}$ is non empty, choose an agent $i$ in $J_{1}$. There is an object $\beta_{0}$ which is in $\sigma(i)$ and not in $\rho(i)$, thus which is in $\rho\left(j_{0}\right)$ for some agent $j_{0}$. If $j_{0}$ is in $J_{3}$, transfer $\beta_{0}$ from agent $j_{0}$ to agent $i$ and stop there. If agent $j_{0}$ is in $J_{1} \cup J_{2}$ there is an object $\beta_{1}$ which is in $\sigma\left(j_{0}\right)$ and in $\rho\left(j_{1}\right)$ for some agent $j_{1}$ different from $j_{0}$. Transfer $\beta_{1}$ from $j_{1}$ to $j_{0}$ and continue the procedure until an agent of $J_{3}$ is reached which has to happen since the objects $\beta_{0}, \beta_{1}, \ldots$ can be chosen to be different and some objects must belong to agents of $J_{3}$ which is a non empty set. The same type of equalities/inequalities as in Step 1 show that the new assignment of $\Omega \cup \widetilde{\alpha}$ so obtained is efficient and gives one more object to the agents of $J_{1}$. Transferring objects to these agents must lead in a finite number of step to an efficient allocation of $\Omega \cup \widetilde{\boldsymbol{\alpha}}$ for which the set $J_{1}$ is empty.

Step 3: Let $\mathrm{p}$ be an efficient assignment of $\Omega \cup \widetilde{\alpha}$, which, by Step 2, can be chosen such that $|\rho(i)| \geq|\sigma(i)|$ for all $\mathrm{i}$ (and also such that $\rho(i) \in \Omega$ for all i). Let $\bar{i}$ be the agent who has $a$ under $a$. Suppose that $\bar{i}$ does not have $a$ under p. Then, since $|\rho(\bar{i})| \geq|\sigma(\bar{i})|$ there exists an object $\beta$ in $\rho(\bar{i})$ which is not in $\sigma(\bar{i})$ and is thus in $\sigma\left(i_{1}\right)$ for some agent $i_{1}$. If $a$ (or $\left.\tilde{\alpha}\right) \in \rho\left(i_{1}\right)$ then exchange $a$ and $\beta$, 
i.e. give a (or $\tilde{\alpha}$ ) to $\bar{i}$ and $\beta$ to $i_{1}$. By Lemma 3.2 (with $C=\rho(\bar{i}) \backslash \beta, \mathrm{D}=\sigma(\bar{i}) \backslash \alpha$ for agent $\bar{i}, \mathrm{C}=\rho\left(i_{1}\right) \backslash \alpha, D^{\prime}=\sigma\left(i_{1}\right) \backslash \beta$ for agent $\left.i_{1}\right)$

$$
\begin{gathered}
V(\bar{i}, \rho(\bar{i}) \backslash \beta \cup \alpha)-V(i, \rho(\bar{i}))=V(i, \sigma(\bar{i}))-V(\bar{i}, \sigma(\bar{i}) \cup \beta \backslash \alpha) \\
V\left(i_{1}, \rho\left(i_{1}\right) \cup \beta \backslash \alpha\right)-V\left(i_{1}, \rho\left(i_{1}\right)\right)=V\left(i, \sigma\left(i_{1}\right)\right)-V\left(i_{1}, \sigma\left(i_{1}\right) \backslash \text { I } \mathrm{U} \text { a }\right)
\end{gathered}
$$

Adding this equalities and exploiting the optimality of $a$ leads to

$$
V(\bar{i}, \rho(\bar{i}) \backslash \beta \cup \alpha)+V\left(i_{1}, \rho\left(i_{1}\right) \cup \beta \backslash \alpha\right) \geq V(i, \rho(\bar{i}))+V\left(i_{1}, \rho\left(i_{1}\right)\right)
$$

so that the new assignment is as efficient as $\mathrm{p}$.

If $\alpha \notin \rho\left(i_{1}\right)$ continue following the objects which are assigned in the assignment $\rho$ differently than in $\mathbf{a}$ : there exists $\beta_{\mathbf{1}}$ in $\rho\left(i_{1}\right)$ which is not in $\sigma\left(i_{1}\right)$ and thus which is in $\sigma\left(i_{2}\right)$ for $i_{2} \# i_{1}$. Either $a$ is in $\rho\left(i_{2}\right)$ in which case the procedure stops, or there exists $\beta_{2}$ in $\rho\left(i_{2}\right)$ and not in $\sigma\left(i_{2}\right)$, so that $\beta_{2}$ is in $\sigma\left(i_{3}\right) \ldots$ Ncyte that even if the same agent is selected several times, the objects $\beta, \beta_{1}, \beta_{2}, \ldots$ can be chosen to be different since, each time an object is in $\sigma(i)$ and not in $\rho(i)$ for some agent $\mathrm{i}$ it must have been replaced by a different object. Since there are a finite number of different objects, at some point an agent $i_{m}$ must be reached such that a (or $\tilde{\alpha})$ is in $\rho\left(i_{m}\right)$. Then replace $\beta$ by $a$ for agent $\bar{i}, \beta_{1}$ by $\beta$ for agent $i_{1}, \ldots, \alpha$ by $\beta_{m-1}$ for agent $i_{m}$. As explained in Stej 1 ihe procedure can always be modified so that agents $\bar{i}, i_{1}, \ldots, i_{m}$ are all different. The same type of equalities than in the simple case where $\mathrm{m}=1$ considered above, combined with the optimality of $\sigma$, implies that the assignment so obtained is as efficient as p.

Remark 1. The property proved in Step 3, namely that the agent $\bar{i}$ who is assigned a under a also receives a under $\mathrm{p}$, justifies the interpretation of $p_{m}(\alpha)$ as the value of a in its second best use: $\mathrm{p}$ attributes the object a to the agent $\bar{i}$ 
who has it under $\sigma$ and attributes the second copy of a to the agent who, after $\bar{i}$, would benefit most of consuming a - perhaps after a reallocation of the other objects.

We now prove that $p^{M}$ and $p_{m}$ are equilibrium prices supporting the efficient allocations of the objects $R$.

\section{2. $p^{M}$ supports the efficient assignments of $\Omega$ to $I$}

Lemma 3.4. Let $a$ be an efficient assignment of $\Omega$ among $\mathbf{I}$. For all $i \in \mathbf{I}$

(i) if $a \in \sigma(i)$, then $p^{M}(\alpha) \leq V(i, \sigma(i))-V(i, \sigma(i) \backslash \alpha)$

(ii) if $\beta \notin \sigma(i)$, then $p^{M}(\beta) \geq V(i, a(i \cup \beta)-V(i, a(i))$

Proof. (i) Let $\alpha \in \sigma(i)$, then $U(\Omega \backslash \alpha) \geq \sum_{j \in I, j \neq i} V(j, \sigma(j))+V(i, \sigma(i) \backslash \alpha)$. Since $p^{M}(\alpha)=U(\Omega)-U(\Omega \backslash \alpha)$,

$p^{M}(\alpha) \leq \sum_{j \in I} V(j, \sigma(j))-\sum_{j \in I, j \neq i} V(j, \sigma(j))-V(i, \sigma(i) \backslash \alpha)=V(i, \sigma(i))-V(i, \sigma(i) \backslash \alpha)$

(ii) Let $\beta \notin \sigma(i)$, and suppose that

$$
p^{M}(\beta)=U(\Omega)-U(\Omega \backslash \beta)<V(i, \sigma(i) \cup \beta)-V(i, \sigma(i))
$$

then,

$$
U(\Omega)<U(\Omega \backslash \beta)+V(i, \sigma(i) \cup \beta)-V(i, \sigma(i))
$$

By Lemma 3.3 there exists an assignment, $\tau$, of $\Omega \backslash \beta$ among $\mathbf{I}$ such that $|\tau(i)| \leq|\sigma(i)|$, and by submodularity and Assumption C

$$
V(i, \sigma(i) \cup \beta)-V(i, \sigma(i)) \leq V(i, \tau(i) \cup \beta)-V(i, \tau(i))
$$

Then,

$$
U(\Omega)<U(\Omega \backslash \beta)+V(i, \tau(i) \cup \beta)-V(i, \tau(i)) I U(\Omega)
$$


which is a contradiction.

Lemma 3.5. For all $i \in I$, there is $A \in P(\Omega)$ such that $|A|=|\sigma(i)|$ and $A \in D\left(i, p^{M}\right)$.

Proof. (a) Suppose that there is $\mathrm{B} \in D\left(i, p^{M}\right)$ such that $|B|<|\sigma(i)|$. From part $(i)$ of Lemma 3.4 we know that $p^{M}(a) \leq V(i, \sigma(i))-V(i, \sigma(i) \backslash \alpha)$ for all $a \in \sigma(i)$. Since $|B|<|\sigma(i)|$, if $a \notin B, p^{M}(\alpha) \leq V(i, B \cup a)-V(i, B)$ so that $B \cup a \in D\left(i, p^{M}\right)$. Following this process, we can add elements to $B$ and obtain $a$ set $B^{*}$ such that $\left|B^{*}\right|=|\sigma(i)|$ and $B^{*} \in D\left(i, p^{M}\right)$.

( $b$ )Suppose that there is $B \in D\left(i, p^{M}\right)$ such that $|B|>|\sigma(i)|$. Then by part (ii) of Lemma 3.4 we know that for all $\beta \in$ Bsuch that $\beta \notin \sigma(i), p^{M}(\beta) \geq V(i, \sigma(i) \cup \beta)-$ $V(i, \sigma(i))$. This implies, since $|B|>|\sigma(i)|$, that $p^{\prime \prime}(\beta) \geq V(i, B)-V(i, B \backslash \beta)$. Since $B \in D\left(i, p^{M}\right)$, it must be that $p^{M}(\beta)=V(i, B)-V(i, B \backslash \beta)$, so that $B \backslash \beta \in D\left(i, p^{M}\right)$. Following this process, we can subtract elements from $B$ and obtain a set $B^{*}$ such that $\left|B^{*}\right|=|\sigma(i)|$ and $B^{*} \in D\left(i, p^{M}\right)$.

Theorem 3.6. If $a$ is an efficient assignment of $\Omega$ among $I$, then $p^{M}$ supports $\sigma$.

Proof: In order to prove the theorem, we must prove that if $\sigma$ is an efficient assignment of the objects, then for all $i \in I, \sigma(i) \in D\left(i, p^{M}\right)$. By Lemma 3.5, there exist $A \in D\left(i, p^{M}\right)$ such that $|A|=|\sigma(i)|$. Suppose that $A \neq \sigma(i)$. Then there exists $\beta \in \sigma(i)$ such that $\beta \notin A$ and there is $a \in A$ such that $a \notin \sigma(i)$. Let us show that $A \cup \beta \backslash \alpha$ is also in $D\left(i, p^{M}\right)$.

Suppose this is not true. Then it must be that $V(i, A)-p^{M}(A)>V(i, A U$ $\beta \backslash \alpha)-P^{M}(A \cup \beta \backslash \alpha)$, which is equivalent to $p^{M}(\beta)-p^{M}(\alpha)>V(i, A \cup \beta \backslash \alpha)-$ 
$V(i, A)$. By Lemma 3.2 (with $C=A \backslash \alpha$ and $D=\sigma(i) \backslash \beta$ ) this is equivalent to

$$
U(\Omega \backslash \alpha)-U(\Omega \backslash \beta)>V(i, \sigma(i))-V(i, \sigma(i) \cup \alpha \backslash \beta)
$$

To show that his inequality is impossible, consider an efficient assignment $\tau$ of $R a$ such that $|\tau(j)| \leq|\sigma(j)|$ for all. $j \in \mathbf{I}$. By Lemma 3.3 such an assignment exists. In order to contradict inequality (3.1) we construct an assignment of $\Omega \backslash \beta$ from $\tau$ by removing the object $\beta$ from the agent who has it under $\tau$ and "appropriately" assigning the object $a$.

Consider the agent $j_{1}$ who receives $\beta$ under $\tau$. If $j_{1}=i$, then take $\beta$ from agent $i$ and replace it by $o$. If $j_{1} \# i$ then since $\beta \in \tau\left(j_{1}\right)$ and $\beta \notin \sigma\left(j_{1}\right)$, and since $\left|\tau\left(j_{1}\right)\right| \leq\left|\sigma\left(j_{1}\right)\right|$, there is an object $\beta_{1}$ in $\sigma\left(j_{1}\right)$ which is not in $\tau\left(j_{1}\right)$. If this object is either $\alpha$ or is such that it belongs to $\tau(i)$, then the procedure stops: in the first case replace $\beta$ by $a$ in the assignment of agent $j_{1}$, in the second replace $\beta$ by $\beta_{1}$ for agent $j_{1}$ and replace $\beta_{1}$ by $\alpha$ for agent $i$. If $\beta_{1}$ cannot be either $a$ or an object of $\tau(i)$, then there exists an agent $j_{2}$ such that $\beta_{1} \in \tau\left(j_{2}\right)$. By the same reasoning, since $\beta_{1} 4 \sigma\left(j_{2}\right)$ there exist an object $\beta_{2}$ in $\sigma\left(j_{2}\right)$ which is not in $\tau\left(j_{2}\right)$. If either this object is a or if it belongs to $\tau(i)$, then procedure stops by replacing $\beta$ by $\beta_{1}$ for agent $j_{1}$, and $\beta_{1}$ by $a$ for agent $j_{2}$ in the first case,by $\beta_{2}$ in the second case and $\beta_{2}$ by $a$ for agent $i$; otherwise it continues. As long as the procedure continues the objects $\beta_{1}, \beta_{2}, \ldots$, can 'be chosen so as to be different from each other since each time that an object in $\tau(j)$ and not in $\sigma(j)$, since $|\tau(j)| \leq|\sigma(j)|$, there is a corresponding object in $\sigma(j)$. Since there is a finite number of objects the procedure must end finding an agent $j_{m}$ such that there exists an object $\beta_{m}$ in $\sigma\left(j_{m}\right)$ which is either a or is such that $\beta_{m} \in \tau(i)$. in the first case consider the assignment of $\Omega \backslash \beta$ such that $\beta$ is repllaced by $\beta_{1}$ for agent $j_{1}, \beta_{1}$ is replaced by $\beta_{2}$ for agent $j_{2}, \ldots$, and $\beta_{m-1}$ is replaced $\mathrm{k}_{j} \alpha$ for agent $j$ In the second case $\beta$ is replaced by $\beta_{1}$ for agent $j_{1}, \beta_{1}$ is replaced by $\beta_{2}$ for agent $j_{2}, \ldots, \beta_{m-1}$ is 
replaced by $\beta_{m}$ for agent $j_{m}$ and $\beta_{m}$ is replaced by $a$ for agent i. Note that the agents $j_{1}, \ldots, j_{m}$ can be chosen so as to be different from each other since, if the same agent $j_{\ell}$ is chosen twice, i.e. if for some $\ell \geq 1, r \geq 1, j_{\ell}=j_{\ell+r}$, then the object $\beta_{\ell+r}$ can directly be chosen in $\sigma\left(j_{\ell}\right)$ instead of $\beta_{\ell}$ the first time that agent $j_{\ell}$ is selected. We now use the assignment just constructed to find a bound on the difference $U(\Omega \backslash \alpha)-C(\Omega \backslash \beta)$. Consider the first case where $\beta_{m}=\mathbf{a}$.

$$
\begin{aligned}
U(\Omega / \alpha)-U(\Omega \backslash \beta) \leq & V\left(j_{1}, \tau\left(j_{1}\right)\right)-V\left(j_{1}, \tau\left(j_{1}\right) \backslash \beta \cup \beta_{1}\right) \\
& +V\left(j_{2}, \tau\left(j_{2}\right)\right)-V\left(j_{2}, \tau\left(j_{2}\right) \backslash \beta_{1} \cup \beta_{2}\right) \\
& +\ldots \\
& \left.+V\left(j_{m}, \tau\left(j_{m}\right)\right)-V\left(j_{m}, \tau\left(j_{m}\right) \backslash \beta_{m-1}\right\rangle_{\lrcorner} \alpha\right)
\end{aligned}
$$

By Lemma 3.2 (with $C=\tau\left(j_{\ell}\right) \backslash \beta_{\ell-1}$ and $D=\sigma\left(j_{\ell}\right) \backslash \beta_{\ell}$ for $\ell=1, \ldots, m$ )

$$
\begin{aligned}
U(\Omega / \alpha)-U(\Omega \backslash \beta) \leq & V\left(i, \sigma\left(j_{1}\right) \backslash \beta_{1} \cup \beta\right)-V\left(j_{1}, \sigma\left(j_{1}\right)\right) \\
& +V\left(j_{2}, \sigma\left(j_{2}\right) \backslash \beta_{2} \cup \beta_{1}\right)-V\left(j_{2}, \sigma\left(j_{2}\right)\right) \\
& +\ldots \\
& +V\left(j_{m}, \sigma\left(j_{m}\right) \backslash \alpha \cup \beta_{m-1}\right)-V\left(j_{m}, \sigma\left(j_{m}\right)\right)
\end{aligned}
$$

By the efficiency of the assignment $a$

$$
\begin{aligned}
V(i, \sigma(i))+V\left(j_{1}, \sigma\left(j_{1}\right)+\cdots+V\left(j_{m}, \sigma\left(j_{m}\right)\right) \geq\right. & V(i, \sigma(i) \cup \alpha \backslash \beta) \\
& +V\left(j_{1}, \sigma\left(j_{1}\right) \backslash \beta_{1} \cup \beta\right)+.- \\
& +V\left(j_{m}, \sigma\left(j_{m}\right) \backslash \alpha \cup D m-l\right)
\end{aligned}
$$

which, combined with the previous inequality implies 


$$
U(\Omega / \alpha)-U(\Omega \backslash \beta) \leq V(i, \sigma(i))-V(i, \sigma(i) \backslash \beta \cup \alpha)
$$

and contradicts (3.1). The proof for the case $\beta_{m} \in \tau(i)$ is similar and left to the reader. Note that it covers, with $\mathrm{m}=0$, the case where $\beta \in \tau(i)$.

Thus inequality (3.1) is impossible so that if $A \in D\left(i, p^{M}\right)$ is different from $\sigma(i)$ then each object of $\boldsymbol{A}$ which is not in $\sigma(i)$ can be replaced by a corresponding object of $\sigma(i)$ and the new subset obtained in this way is still in the demand of A. After a finite number of such replacements the subset $\sigma(i)$ will be obtained, so that $\sigma(i) \in D\left(i, p^{M}\right)$.

\section{3. $p_{m}$ supports the efficient assignments of $\Omega$ to I}

Lemma 3.7. Let $a$ be an efficient assignment of $\Omega$ among $I$. For all $i \in I$

(i) if $a \in \sigma(i)$, then $p_{m}(\alpha) \leq V(i, \sigma(i))-V(i, \sigma(i) \backslash \alpha)$

(ii) if $\beta \notin \sigma(i)$, then $p_{i, 2}(\beta) \geq V(i, \sigma(i) \cup \beta)-V(i, \sigma(i))$

Proof. (i) Since we have proved that $p^{M}$ is an equilibrium price, Proposition 3.1 implies that $p_{m} \leq p^{M}$. Since, by Lemma $3.4, p^{M}$ satisfies the inequality (i), so does $p_{m}$.

(ii) If $\beta \notin \sigma(i)$, adding $\beta$ to the objects of $i$ creates an assignment of $\Omega \cup \widetilde{\beta}$. Thus $U(\Omega \cup \widetilde{\beta}) \geq U(\Omega)-V(i, \sigma(i))+V(i, \sigma(i) \cup \beta)$, which is equivalent to the inequality in (ii).

Lemma 3.8. For all $i \in I$, there is $A \in P(\Omega)$ such that $|A|=|\sigma(i)|$ and $A \in D\left(i, p_{m}\right)$.

Proof. The proof is identical to the proof of Lemma 3.5.

Theorem 3.9. If $\sigma$ is an efficient assignment of $\Omega$ among $I$, then $p_{m}$ supports $a$. 
Proof. We must prove that for all $i, \sigma(i) \in D\left(i, p_{m}\right)$. ByLemma3.8, there exists $\boldsymbol{A}$ in the demand of agent $i$ such that $|A|=|\sigma(i)|$. Let us show that if $\boldsymbol{A} \neq \sigma(i)$, then every object $\alpha$ in $\boldsymbol{A}$ and not in $\sigma(i)$ can be replaced by an object $\beta$ in $\sigma(i)$ and not in $A$, so that $A \cup \beta \backslash \alpha$ is in the demand of agent i. By the same reasoning than in the proof of Theorem 3.6, if $\boldsymbol{A} U \beta \backslash \alpha$ were not in the demand of agent $\mathrm{i}$, then the following inequality would have to hold

$$
U(\Omega \cup \widetilde{\beta})-U(\Omega \cup \widetilde{\alpha})>V(i, \sigma(i))-V(i, \sigma(i) \cup \alpha \backslash \beta)
$$

To show that this equality is impossible, choose an efficient assignment $\mathrm{p}$ of $\Omega \cup \widetilde{\beta}$ such that $\rho(j) \in \Omega,|\rho(j)| \geq|\sigma(j)|$, for all $j \in \mathbf{I}$ and such that $\beta \in \rho(i)$. By Lemma 3.3 such an assignment exists. There are two possible cases:

Case 1: $\mathrm{p}$ assigns $\beta$ and not $a$ to agent i. Ther considers the assigr.ment of $\Omega \cup \tilde{\alpha}$ obtained in replacing $\beta$ by cr.

$U(\Omega \cup \widetilde{\alpha}) \geq U(\Omega \cup \widetilde{\beta})-V(i, \rho(i))+V(i, \rho(i) \backslash \beta \cup \alpha)=U(\Omega \cup \widetilde{\beta})-V(i, \sigma(i))+V(i, \sigma(i) \backslash \beta \cup \alpha)$

where the last equality follows from Lemma 3.2 with $C=\rho(i) \backslash \beta, \mathrm{D}=\sigma(i) \backslash \beta$. This contradicts inequality (3.2).

Case 2. p assigns $\beta$ and $\alpha$ to agent i. Let $j$ be the agent who receives $\alpha$ under $\sigma$, and let $k$ be the agent who receives the copy $\widetilde{\beta}$ of $\beta$ under $\mathrm{p}$. If $\mathbf{j}=\mathrm{k}$, then take $\widetilde{\beta}$ from agent $\mathbf{j}$ and replace it by 6 .

$U(\Omega \cup \widetilde{\beta})-U(\Omega \cup \widetilde{\alpha}) \leq V(j, \rho(j))-V(j, \rho(j) \cup \alpha \backslash \beta)=V(j, \sigma(j) \cup \beta \backslash \alpha)-V(j, \sigma(j))$ where the last equality follows from Lemma 3.2 with $\mathrm{C}=\rho(j) \backslash \beta$ and $\mathrm{D}=\sigma(j) \backslash \alpha$. By efficiency of the assignment a,

$$
V(j, \sigma(j) \cup \beta \backslash \alpha)-V(j, \sigma(j)) \leq V(i, \sigma(i))-V(i, \sigma(i) \cup \alpha \backslash \beta)
$$


This contradicts inequality (3.2). If $\mathbf{j} \neq h$, then since $a \in \sigma(j), a \notin \rho(j)$, and $|\rho(j)| \geq|\sigma(j)|$, there is an object $\beta_{0}$ in $\rho(j)$ which is not in $\sigma(j)$. If this object $\beta_{0}$ belongs to $\sigma(k)$ or $\sigma(i)$, then the procedure stops: in the first case replace $\beta_{0}$ by $\widetilde{\alpha}$ for agent $j$, and replace $\widetilde{\beta}$ by $\beta_{0}$ for agent $\mathrm{k}$. In the second case replace $\beta_{0}$ by $\widetilde{\alpha}$ for agent $\mathrm{j}$, and replace $\beta$ by $\beta_{0}$ for agent $\mathrm{i}$. If $\beta_{0}$ is neither in $\sigma(k)$ nor in $\sigma(i)$, then there is an agent $j_{1}$ such that $\beta_{0} \in \sigma\left(j_{1}\right)$. By the same reasoning, since $\beta_{0} \notin \rho\left(j_{1}\right)$, and $\left|\rho\left(j_{1}\right)\right| \geq\left|\sigma\left(j_{1}\right)\right|$, there is an object $\beta_{1}$ in $\rho\left(j_{1}\right)$ which is not in $\sigma\left(j_{1}\right)$. If $\beta_{1}$ belongs to $\sigma(k)$ or $\sigma(i)$, then the procedure stops by replacing $\beta_{0}$ by $\widetilde{\alpha}$ for agent $j, \beta_{1}$ by $\beta_{0}$ for agent $j_{1}$, and $\widetilde{\beta}$ by $\beta_{1}$ for agent $\mathrm{k}$ in the first case, and $\beta$ by $\beta_{1}$ for agent $\mathrm{i}$ in the second case; otherwise it continues. As long as the procedure continues the objects $\beta_{1}, \beta_{2}, \ldots$, can be chosen so as to be different from each other since each time that an object in $\sigma(l)$ is not in $\rho(l)$, and since $|\rho(l)| \geq|\sigma(l)|$, there is a corresponding object in $\rho(l)$. Since there is a finite number of objects the procedure must end finding an agent $j_{m}$ such that $\beta_{m} \in \rho\left(j_{m}\right)$, and $\beta_{m} \notin \sigma\left(j_{m}\right)$, and such that $\beta_{m}$ is in $\sigma(k)$ or $\sigma(i)$. In the first case consider the assignment of $\Omega \cup \widetilde{\alpha}$ such that $\beta_{0}$ is replaced by $\widetilde{\alpha}$ for agent $j, \beta_{1}$ is replaced by $\beta_{0}$ for agent $j_{1}, \beta_{2}$ is replaced by $\beta_{1}$ for agent $j_{2}, \ldots . ., \beta_{m}$ is replaced by $\beta_{m-1}$ for agent $j_{m}$, and $\widetilde{\beta}$ is replaced by $\beta_{m}$ for agent $\mathrm{k}$. In the second case $\beta_{0}$ is replaced by $\widetilde{\alpha}$ for agent $\mathbf{j}, \beta_{1}$ is replaced by $\beta_{0}$ for agent $j_{1}, \beta_{2}$ is replaced by $\beta_{1}$ for agent $j_{2}, \ldots ., \beta_{m}$ is replaced by $\beta_{r,-1}$ for agent $j_{m}$, and $\beta$ is replaced by $\beta_{m}$ for agent i. Note that, as in the proofs of Lemma 3.3 and Theorem 3.6, we can assume w.l.o.g. that the agents $j_{1}, \ldots, j_{m}$ are all different.

We now use the assignment just constructed to find a bound on the difference $\mathrm{U}(\mathrm{O} \cup \widetilde{\beta})-\mathrm{U}(\mathrm{O} \cup 6)$. Consider the case where $\beta_{m} \in \sigma(i)$.

$$
\begin{aligned}
U(\Omega \cup \widetilde{\beta})-U(\Omega \cup \widetilde{\alpha}) \leq & V(j, \rho(j))-V\left(j, \rho(j) \cup \alpha \backslash \beta_{0}\right) \\
& +V\left(j_{1}, \rho\left(j_{1}\right)\right)-V\left(j_{1}, \rho\left(j_{1}\right) \cup \beta_{0} \backslash \beta_{1}\right)
\end{aligned}
$$




$$
\begin{aligned}
& +\ldots \\
& +V\left(j_{m}, \rho\left(j_{m}\right)\right)-V\left(j_{m}, \rho\left(j_{m}\right) \cup \beta_{m-1} \backslash \beta_{m}\right) \\
& +V(i, \rho(i))-V\left(i, \rho(i) \cup \beta_{m} \backslash \beta\right)
\end{aligned}
$$

By Lemma 3.2 (with $C=\rho\left(j_{l}\right) \backslash \beta_{l}$ and $D=\sigma\left(j_{l}\right) \backslash \beta_{l-1}$ for $l=0,1, \ldots, m$ where $j_{0}=j$, and $\beta_{0-1}=a$, and $C^{\prime}=\rho(i) \backslash \beta, D^{\prime}=\sigma(i) \backslash \beta \backslash \beta_{m} u$ a $)$

$$
\begin{aligned}
U(\Omega \cup \widetilde{\beta})-U(\Omega \cup \widetilde{\alpha}) \leq & V\left(j, \sigma(j) \cup \beta_{0} \backslash \alpha\right)-V(j, \sigma(j)) \\
& +V\left(j_{1}, \sigma\left(j_{1}\right) \cup \beta_{1} \backslash \beta_{0}\right)-V\left(j_{1}, \sigma\left(j_{1}\right)\right) \\
& +\ldots \\
& +V\left(j_{m}, \sigma\left(j_{m}\right) \cup \beta_{m} \backslash \beta_{m-1}\right)-V\left(j_{m}, \mid \sigma\left(j_{m}\right)\right) \\
& +V\left(i, \sigma(i) \cup \alpha \backslash \beta_{m}\right)-V(i, \sigma(i)) \\
& +V(i, \sigma(i))-V(i, \sigma(i) \cup \alpha \backslash \beta)
\end{aligned}
$$

By the efficiency of the assignment a

$$
\begin{gathered}
V(j, \sigma(j))+\ldots+V\left(j_{m}, \sigma\left(j_{m}\right)\right)+V(i, \sigma(i)) \geq \\
V\left(j, \sigma(j) \cup \beta_{0} \backslash \alpha\right)+\ldots+V\left(j_{m}, \sigma\left(j_{m}\right) \cup \beta_{m} \backslash \beta_{m-1}\right)+V\left(i, \sigma(i) \cup \alpha \backslash \beta_{m}\right)
\end{gathered}
$$

which, combined with the previous inequality implies

$$
U(\Omega \cup \widetilde{\beta})-U(\Omega \cup \widetilde{\alpha}) \leq{ }^{\top}(i, \sigma(i))-V(i, \sigma(i) \cup \alpha \backslash \beta)
$$

and contradicts inequality (3.2). Thus (3.2) is impossible so that if $A \in D\left(i, p_{m}\right)$ is different from $\sigma(i)$ then each object of $\boldsymbol{A}$ which is not in $\sigma(i)$ can be replaced by a corresponding object of $\sigma(i)$, and the new subset obtained in this way is still in the demand of A. After a finite number of such replacements the subset $\sigma(i)$ will be obtained, so that $\sigma(i) \in D\left(i, p_{m}\right)$. The case where $\beta_{m}$ belong to $\sigma(k)$ is similar and left to the reader. 


\subsection{The lattice structure of equilibrium prices}

Theorem 3.10. The set of prices supporting the efficient assignments of $\Omega$ is a convex, complete lattice.

Proof. The set of prices supporting an efficient assignment $\sigma$ of $\Omega$ is the set of solutions to the linear inequalities $V(i, \sigma(i))-p(\sigma(i)) \geq V(i, \mathrm{~A})-p(A), \quad \mathrm{VA} \in \Omega$ and is thus closed and convex. To prove the theorem, we thus only need to prove that if $\mathrm{p}$ and $p^{\prime}$ are two prices supporting an efficient assignment $\mathbf{a}$ of $\Omega$ then p A $p^{\prime}$ and $\mathrm{p} \vee p^{\prime}$, defined by $\mathrm{p} \mathrm{A} p^{\prime}(\alpha)=\min \left\{p(\mathrm{a}), p^{\prime}(\mathrm{a})\right)$ and $\mathrm{p} \vee p^{\prime}(\mathrm{a})=$ $\max \left\{p(\alpha), p^{\prime}(\mathrm{a})\right)$ for all $a \in \Omega$, also support a. This amouncs to showing that $\sigma(i) \in D\left(i, p \mathrm{Ap}^{\mathrm{t}}\right)$ and $\sigma(i) \in D\left(i, \ddot{p} \vee p^{\prime}\right)$ for all $\mathrm{i} \in \mathbf{I}$. First note that since, by Proposition 3.1, $p_{m} \leq \mathrm{p} \mathrm{A} p^{\prime} \leq \mathrm{p} \vee p^{\prime} \leq p^{M}$, the inequalities (i) and (ii) of Lemma 3.4 or 3.7 are satisfied by $p \wedge p^{\prime}$ and $p \vee p^{\prime}$. By the same reasoning as in Lemma 3.5, this implies that, for all $\mathrm{i}$ there exist $A_{i}$ in $D\left(i, \mathrm{p} \wedge p^{\prime}\right)$ and $A_{i}^{\prime}$ in $D\left(i, \mathrm{p} \vee \mathrm{p}^{\prime}\right)$ such that $\left|A_{i}\right|=\left|A_{i}^{\prime}\right|=|\sigma(i)|$. Suppose that, for some agent i, $A_{i} \neq \sigma(i)$. Then there exists $a$ such that $\alpha \in A_{i}$ and $\alpha \notin \sigma(i)$, and there exists $\beta$ such that $\beta \in \sigma(i)$ and $\beta \notin \mathrm{A}$,. Let us show that $A_{i} \backslash \alpha \cup \beta$ is also in $D\left(i, p \wedge p^{\prime}\right)$. By Lemma 3.2 (with $C=A \backslash \alpha$ and $\mathrm{D}=\sigma(i) \backslash \beta)$ and the fact that $\mathrm{p}$ and $p^{\prime}$ support $a$

$$
\begin{aligned}
V\left(i, A_{i} \backslash \alpha \cup \beta\right)-V\left(i, A_{i}\right) & =V(i, \sigma(i))-V(i, \sigma(i) \backslash \beta \cup \alpha) \\
& \geq \max \left\{p(\alpha)-p(\beta), p^{\prime}(\alpha)-p^{\prime}(\beta)\right\} \\
& \geq \max \left\{p \vee p^{\prime}(\alpha)-p \vee p^{\prime}(\beta), p \vee p^{\prime}(\alpha)-p \vee p^{\prime}(\beta)\right\}
\end{aligned}
$$

where the last inequality can easily be checked case by case. Thus the objects of $A_{\boldsymbol{i}}$ which are not in $\sigma(i)^{Y}$ can be replaced by objects of $\sigma(i)$, which proves that $\sigma(i) \in D\left(i, p \wedge p^{\prime}\right)$. The same reasoning applied to $A_{i}^{\prime}$ shows that $\sigma(i) \in D\left(i, p \vee p^{\prime}\right)$. Thus the set of prices supporting the assignment $a$ is a lattice, and being closed, it is complete. 
Note that choosing prices independently for each object a between $p^{M}(\alpha)$ and $p_{m}(\alpha)$ does not generally lead to a vector of equilibrium prices, as shown by the following example:

Example 3.11. : Let e $\in \mathcal{E}$ be such that $\mathbf{I}=\{1,2,3\}, \Omega=\{\alpha, \beta, \gamma\}$. The reservation values of the agents for the different subsets of objects are given in the following table:

\begin{tabular}{|l|l|l|l|l|l|l|l|}
\hline $\mathrm{V} \backslash \mathrm{A}$ & $\alpha$ & $\beta$ & $\gamma$ & $\alpha \beta$ & $\alpha \gamma$ & $\beta \gamma$ & $\alpha \beta \gamma$ \\
\hline$V(1, A)$ & 8 & 9 & 8 & 16 & 15 & 16 & 22 \\
$V(2, A)$ & 3 & 7 & 6 & 9 & 8 & 12 & 13 \\
$V(3, A)$ & 5 & 4 & 7 & 8 & 11 & 10 & 13 \\
\hline
\end{tabular}

For this economy the efficient assignment is $\sigma(1)=\{\alpha \beta\}, \sigma(2)=\emptyset, \sigma(3)=\{\mathrm{y})$.

The vectors $p^{M}$ and $p_{m}$ are

$$
p^{M}=(7,8,7), \quad p_{m}=(4,7,6)
$$

The price vector $\mathrm{p}=(4,7,7)$ however is not an equilibrium price vector since at these prices agent $\mathbf{3}$ would demand object $a$ and not object $\mathrm{y}$. The prices of objects need to be compatible: in particular the surplus of agent 3 on object $\gamma$ has to be as least as large as on objects a. The set of equilibrium prices is $\{(4+\varepsilon, 7, p(\gamma)) \mid 6 \leq p(\gamma) \leq \min (6+\varepsilon, 7)), 0 \leq \varepsilon \leq 3\}$

\section{Relation between the Cardinality Condition and Gross Substi- tutability}

We mentioned in the Introduction and in Section 2 that, for submodular reservation values, the Cardinality Condition implies that agents' demands satisfy the 
Gross Substitute property. We now formally prove this claim. The proof uses the following properties of agents' demands when their reservation value functions satisfy submodularity and the Cardinality Condition.

Lemma 4.1. Suppose that the reservation value $V(i, \cdot)$ of agent $i$ is submodular and satisfies the Cardinality Condition. Then

(i) If $A$ and $B$ are two subsets of $D(i, p)$, and if $|B|<|A|$, then for every $a$ such that $a \in A, a \notin B$, then $B \cup a$ is in $D(i, p)$

(ii) If $p$ and $p^{\prime}$ are two vectors of prices such that $p^{\prime} \geq \mathrm{p}$, if $A$ is a subset of $D(i, p)$ of maximum cardinality, then for all $B \in D\left(i, p^{\prime}\right),|B| \leq|A|$.

Proof. (i) $|B|<|A|$ implies $|B U \alpha| \leq|A|$ so that $V(i, \mathrm{~B} \cup a)-V(i, B) \geq$ $V(i, A)-V(i, A \backslash \alpha) \geq p(a)$, where the last inequaiity comes from the fact that $A \in D(i, p)$. Thus the surplus of agent $i$ with the objects of $B \mathrm{U} a$ is at least as large as with the objects of $B$, so that $B \cup a \in D(i, p)$.

(ii) Suppose $|B|>|A|$. Then there exists $\beta$ such that $\beta \in B, \beta \notin A$. Since $|A \cup \beta| \leq|B|, V(i, A \cup \beta)-V(i, A) \geq V(i, B \backslash \beta)-V(i, B) \geq p^{\prime}(\beta) 2 p(\beta)$. Thus $A \cup \beta$ is in $D(i, p)$, which contradicts the assumption that $A$ has the maximum number of elements among the subsets of $D(i, p)$.

Proposition 4.2. Suppose that the reservation value $V(i, \cdot)$ of agent $i$ is submodular and satisfies the Cardinality Condition. Then agent $i^{\prime} \mathrm{s}$ demand satisfies the Gross Substitute property.

Proof. Let $p^{\prime}$ be a price vector such that $p^{\prime} \geq p$ and let $a$ be an element of $D(i, p)$ such that $p(\alpha)=p^{\prime}(a)$. By Lemma 4.1 (i), there is a subset $|A|$ of maximum cardinality among the subsets of $D(i, p)$ such that $a \in A$. Let $B \in D\left(i, p^{l}\right)$. By Lemma 4.1 (ii), $|B| \leq|A|$. If $\alpha \notin B$ and $|B|<|A|$, then $V(i, B \cup a)-V(i, B) \geq$ 
$V(i, A)-V(i, A \backslash \alpha) \geq p(\alpha)=p^{\prime}(\alpha)$, so that $\mathrm{B} \cup \alpha \in D\left(i, p^{\prime}\right)$. If $\mathrm{a} \notin B$ and $|A|=|B|$, there exist $\beta \in \mathrm{B}, \beta \notin \mathrm{A}$. By Lemma 3.2, $V(i, B \backslash \beta \cup \alpha)-V(i, \mathrm{~B})=$ $V(i, \mathrm{~A})-V(i, A \backslash \alpha \cup \beta) \geq p(\alpha)-p(\beta) \geq p^{\prime}(\alpha)-p^{\prime}(\beta)$ where the last two inequalities come from the facts that $\mathrm{A}$ is at least as desirable at prices $\mathrm{p}$ than $A \backslash \alpha \cup \beta$, that $p(\alpha)=p^{\prime}(\alpha)$, and $p^{\prime}(\beta) \geq p(\beta)$. Thus $B \backslash \beta \cup \mathrm{a} \in \mathrm{D}\left(\mathrm{i}, \mathrm{p}^{1}\right)$ so that $\mathrm{a} \in D\left(i, p^{\prime}\right)$.

The reverse proposition is not true: the GS property on demand holds for reservation value functions which do not satisfy the Cardinality Condition. For example it holds for the reservation value functions $V(i, \mathrm{~A})=\max \{V(i, \mathrm{a}), \mathrm{a} \in$ $\boldsymbol{A})$, which correspond to the case where agants have use for only one object. In this paper we are interested in the case where the agents are always willing to purchase one more object if the price is sufficiently low, i.e. to situations where the marginal utility of every object is always positive. We have not succeeded in characterizing all reservation value functions which lead to the Gross Substitute property, so that we do not know if they involve functions with a natural economic interpretation, other than the ones satisfying the Cardinality Co/dition. It is clear on simple examples that the Gross Substitute property allow for more "free parameters" in constructing the reservation value functions than the Cardinality Condition. If we come back to the example of Section 2 for which there is no equilibrium, and modify it to obtain existence of an equilibrium, then in order to have the Cardinality Condition satisfied, we can keep the same reservation values for objects $\alpha, \beta, \gamma$ and the reservation values for one of the subsets composed of two objects (for example we keep the numbers in the column $\beta \gamma$ ). Then the choice of numbers in this column determine all other reservation values for groups of two objects (since the marginal contribution of $\mathbf{a}$ and $\gamma$ to one-object subsets are determined). The values for the three-object subset $\alpha \beta \gamma$ are then "free" parameters (subject to the submodularity condition and monotonicity, that we 
have not used in the proofs, but is a natural assumption to require). For example, if we keep the same values for $V(i, \beta \mathrm{U} \gamma)$ as in the original example, then the reservation values table must be

\begin{tabular}{|l|l|l|l|l|l|l|l|}
\hline$V \backslash A$ & $\alpha$ & $\beta$ & $\gamma$ & $\alpha \beta$ & $\alpha \gamma$ & $\beta \gamma$ & $\alpha \beta \gamma$ \\
\hline$V(1, A)$ & 10 & 8 & 2 & 17 & 11 & 9 & $\geq 17$ \\
$V(2, A)$ & 8 & 5 & 10 & 11 & 16 & 13 & $\geq 16$ \\
$V(3, A)$ & 1 & 1 & 8 & 2 & 9 & 9 & $\geq 9$ \\
\hline
\end{tabular}

If we only require that the demands satisfy the Gross Substitute property, then the only restrictions on the agents' reservation values for subsets of two objects are as follows: if $V\left(i, \alpha_{1} \cup \alpha_{2}\right)-V\left(i, \alpha_{2}\right)<V\left(i, \alpha_{1} \cup \alpha_{3}\right)-V\left(i, \alpha_{3}\right)$ then it must be that $V\left(i, \alpha_{1} \mathrm{U} \alpha_{3}\right)-V\left(i, \alpha_{1}\right)=V\left(i, \alpha_{2} \mathrm{U} \alpha_{3}\right)-V\left(i, \alpha_{2}\right)$. For if, for example, we had $V\left(i, \alpha_{1} \cup \alpha_{3}\right)-V\left(i, \alpha_{1}\right)>V\left(i, \alpha_{2} \cup \alpha_{3}\right)-V\left(i, \alpha_{2}\right)$ then for prices $\mathrm{p}$ such that $V\left(i, \alpha_{1} \cup \alpha_{2}\right)-V\left(i, \alpha_{2}\right)<p\left(\alpha_{1}\right)<V\left(i, \alpha_{1} \mathrm{U} \alpha_{3}\right)-V\left(i, \alpha_{3}\right)$, $V^{\prime}\left(i\right.$, a $\left._{2} \cup \alpha_{3}\right)-V\left(i, \alpha_{2}\right)<\mathrm{p}\left(\alpha_{3}\right)<V\left(i, \alpha_{1} \cup \alpha_{3}\right)-V\left(i, \alpha_{1}\right)$ and $V\left(i, \alpha_{2}\right)-p\left(\alpha_{2}\right)=$ $V\left(i, \alpha_{1} \cup \alpha_{3}\right)-p\left(\alpha_{1}\right)-p\left(\alpha_{3}\right)$, agent i 's demand would consist of the sets $\left\{\alpha_{1}, \alpha_{3}\right\}$ and $\left\{\alpha_{2}\right\}$. If the price $p\left(\alpha_{1}\right)$ slightly increases ${ }^{1}$ then the demand reduces to $\left\{\alpha_{2}\right\}$, which violates the Gross Substitute property. A similar reasoning eliminates the possibility that $V\left(i, \alpha_{1} \cup \alpha_{3}\right)-V\left(i, \alpha_{1}\right)<V\left(i, \alpha_{2} \cup \alpha_{3}\right)-V\left(i, \alpha_{2}\right)$. Thus, when the column $V(i, \beta \cup \gamma)$ is chosen, there are stil! some degrees of freedom for choosing the values of $V(i, \mathrm{a} \cup \beta)$ and $V(i, \mathrm{a} \cup \gamma)$ compatible with the GS property of the demand. For example, we could keep the two columns $\boldsymbol{a} 7$ and $\beta \gamma$ of the original

\footnotetext{
${ }^{1}$ in such a way that the inequality $p\left(\alpha_{1}\right)<V\left(i, \alpha_{1} \cup \alpha_{3}\right)-V\left(i, \alpha_{3}\right)$ which ensures that $\alpha_{3}$ is not in the demand, still holds'
} 
table, and the table of numbers has just to be modified so as to satisfy

\begin{tabular}{|l|l|l|l|c|l|l|l|}
\hline$V \backslash A$ & $\alpha$ & $\beta$ & $\gamma$ & $\alpha \beta$ & $\alpha \gamma$ & $\beta \gamma$ & $\alpha \beta \gamma$ \\
\hline$V(1, A)$ & 10 & 8 & 2 & $1 \hat{} \leq V(1, \alpha \cup \beta) \leq 17$ & 11 & 9 & $\geq \max \{11, V(1, \alpha \cup \beta)\}$ \\
$V(2, A)$ & 8 & 5 & 10 & 11 & 14 & 13 & $\geq 14$ \\
$V(3, A)$ & 1 & 1 & 8 & $1 \leq V(3, \alpha \cup \beta) \leq 2$ & 9 & 9 & $\geq 9$ \\
\hline
\end{tabular}

Thus there are more "free" parameters with the GS assumption than with the Cardinality Condition. It would be interesting to characterize all reservation functions which lead to demands satisfying the GS property, in order to find which interpretable restrictions on the preferences of the agents are compatible with the GS property. Hopefully, future research will provide an answer to this question.

\section{References}

[1] Bikhchandani S. and J. Mamer (1994), "Competitive equilibrium in an exchange economy with indivisibilities", mimeo University of California, Los Angeles.

[2] Gale, D. (1984), "Equilibrium in a Discrete Exchange Economy with Money", International Journal of Game Theory, 13, 61-64.

[3] Gul, F. and E. Stacchetti (1996), "Walrasian Equilibrium Without Complementarity", mimeo.

[4] Henry C. (1970), "Indivisibilite dans une Economie d'Echanges", Econometrica Vol. 38, 3, 542-558.

[5] Kaneko, M. (1982), "The Central Assignment Game and the Assignment Markets", Journal of Mathematical Economics, 10, 205-232. 
[6] Kaneko, M. and Y. Yamamoto (1986), "The Existence and Computation of Competitive Equilibria in Markets with an Indivisible Commodity", Journal of Economic Thwry, 38, 118-136

[7] Kelso A.S., and V.P.Crawford (1982), "Job Matching, Coalition Formation, and Gross Substitutes", Econometrica Vol. 50, 6, 1483-1504.

[8] Laan G., D. Talman and Z. Yang (1995), "Existence of an equilibrium in a Competitive Economy of Indivisibilities with money", Tinbergen Institute Discussion Paper TI 8-95-200.

[9] Quinzii, M. (1984), "Core and Competitive Equilibria with Indivisibilities", International Journal of Game Thwry, 13, 41-60.

[10] Roth A. E. and M. A. Oliveira Sotomayor (1990), Two-sided Matching, Cambridge: Cambridge University Press.

[11] Shapley L. S. and M. Shubik (1972), "The assignment game I: The core", International Journal of Game Thwry 1,111-130. 